\title{
Early membrane exposure of phosphatidylserine followed by late necrosis in murine macrophages induced by Candida albicans from an HIV-infected individual
}

\author{
L. A. PANAGIO, I. FELIPE*, M. C. VIDOTTO and L. C. J. GAZIRI† \\ Departments of Microbiology, * General Pathology and †Physiological Sciences, Universidade Estadual de \\ Londrina, 86051-990 Londrina, Brazil
}

\begin{abstract}
The hypothesis that Candida albicans isolate (CR1) from an HIV-infected individual induced apoptosis of macrophages was examined by optical microscopy, binding of annexin V-FITC and analyses of DNA degradation (TUNEL tests and agarose gel electrophoresis). Resident murine peritoneal macrophages co-incubated for 5-15 min with $C$. albicans CR1 bound annexin V, whereas macrophages incubated with either heat-inactivated strain CR1, $C$. albicans 577 (isolated from a patient with mucocutaneous candidiasis) or $C$. albicans FCF14 (a mutant that did not produce proteases and phospholipases) did not bind annexin for up to $2 \mathrm{~h}$ of observation. However, macrophages exposed to $C$. albicans CR1 did not present the pattern of DNA degradation typical of apoptosis. Macrophages became increasingly permeable to propidium iodide from $30 \mathrm{~min}$ to $2 \mathrm{~h}$ after their exposure to $C$. albicans CR1. Most of the phagocytosed $C$. albicans CR1 yeast cells switched to germ-tubes inside the macrophages after incubation for 1-2 h. These results show that macrophages exposed to $C$. albicans CR1 presented early signs of apoptosis but progressed to necrosis, and suggest that Candida strains that readily switch to germ-tubes inside those apoptotic cells might have a competitive advantage in vivo because released germ-tubes resist further attack by macrophages.
\end{abstract}

\section{Introduction}

Following the first demonstration that Shigella flexneri was able to induce macrophage apoptosis in vitro [1], several other bacteria were also shown to induce apoptosis [2-7]. It was usually observed that the bacteria must be phagocytosed and viable to induce apoptosis, although some bacterial proteins and an HIV protein induced apoptosis by themselves [8-11]. Induction of apoptosis by bacterial infection in vivo was also demonstrated and the cell death and ensuing changes in the secretion of cytokines may be important in microbial pathogenesis $[12,13]$. These observations led to the concept that the ability of pathogens to induce apoptosis of phagocytes might be an important virulence factor, for it would curtail the host's defence mechanisms.

Received 15 Jan. 2002; revised version accepted 19 June 2002.

Corresponding author: Professor L. C. J. Gaziri (e-mail: gaziri@uel.br).
Although innate cellular immunity is of prime importance for the host defence against fungal infections, it is not known whether Candida albicans and other fungi can exploit phagocyte apoptosis to their own advantage. Phagocytosis of heat-killed C. albicans increased the apoptosis of human neutrophils after culture for $18 \mathrm{~h}$ [14]. In contrast, infection by $C$. albicans inhibited apoptosis of human monocytes and supernates from co-cultures of neutrophils and $C$. albicans inhibited apoptosis of naive neutrophils [1416].

Previous studies showed that murine peritoneal macrophages phagocytose $C$. albicans in vitro through both complement and mannose receptors and that concanavalin A-activated macrophages have an increased ability to ingest and kill $C$. albicans [17-19]. In preliminary experiments aimed at characterising the ability of macrophages to phagocytose and kill a seemingly more virulent strain of $C$. albicans recently isolated from an HIV-infected individual, this strain caused complete destruction of macrophage mono- 
layers after co-incubation for $2 \mathrm{~h}$; however, when observed after co-incubation for only $30 \mathrm{~min}$ these macrophages had phagocytosed several yeast cells and some of their nuclei showed peripheral condensations resembling those seen at the beginning of apoptosis. Therefore, the possibility that these macrophages might have entered into apoptosis and were subsequently lysed was investigated in the present study.

\section{Materials and methods}

\section{Micro-organisms and cultivation}

C. albicans 577, isolated from the skin of a patient with mucocutaneous candidiasis, C. albicans CR1, isolated from the oral mucosa of an HIV-infected patient at the Dentistry School, Universidade Estadual de Londrina, Brazil, and C. albicans FCF14, a mutant that did not produce proteases and phospholipases (kindly provided by Dr M. T. Shimizu, School of Dentistry, Universidade Estadual Paulista, Botucatu, Brazil) were used in this study. The fungi were maintained at room temperature on Sabouraud dextrose agar; fungal cells were obtained by growth in Sabouraud dextrose broth (Difco) for $24 \mathrm{~h}$ at $28^{\circ} \mathrm{C}$ with agitation $(120 \mathrm{rpm})$. The blastoconidia were harvested by centrifugation (2000 $\mathrm{g}, 6 \mathrm{~min})$, washed three times with phosphate-buffered saline (PBS) and resuspended at $4 \times 10^{6}$ cells $/ \mathrm{ml}$ in RPMI 1640 medium (Sigma) containing bovine serum albumin $1 \%$.

Heat-inactivated C. albicans were used in some experiments and were obtained by incubating $4 \times$ $10^{6}$ cells $/ \mathrm{ml}$ in PBS at $60^{\circ} \mathrm{C}$ for $1 \mathrm{~h}$; samples of these preparations were plated on Sabouraud agar to ascertain that $C$. albicans was inactivated.

C. albicans were opsonised immediately before the phagocytosis experiments by incubating $4 \times 10^{6}$ cells in $1 \mathrm{ml}$ of RPMI medium containing fresh non-immune mouse serum $2.5 \%$ for $5 \mathrm{~min}$ at $37^{\circ} \mathrm{C}$; these cells were then cytospun and resuspended in RPMI medium.

Avian Escherichia coli UEL 17 and Shigella flexneri M90T (kindly supplied by P. J. Sansonetti) were maintained on Luria-Bertani agar and were grown to $10^{8}$ cells $/ \mathrm{ml}$ in $\mathrm{RPMI} 1640$ at $37^{\circ} \mathrm{C}$.

\section{Mouse peritoneal macrophages}

Resident peritoneal phagocytes were collected by rinsing the peritoneal cavity of male Swiss mice (weighing c. $30 \mathrm{~g}$ ) with $3 \mathrm{ml}$ of RPMI 1640 medium containing BSA $1 \%$. The cells were cytospun, resuspended in RPMI medium, counted in a haemocytometer, and the concentration was adjusted to $4 \times$ $10^{5}$ cells $/ \mathrm{ml}$. The cells were allowed to adhere to glass covers for $1 \mathrm{~h}$ at $37^{\circ} \mathrm{C}$ under an atmosphere of $\mathrm{O}_{2} 95 \%$, $\mathrm{CO}_{2} \quad 5 \%$ and the preparation was then rinsed with RPMI 1640 medium to remove non-adherent cells. The cell monolayer adhered to the glass cover contained almost exclusively macrophages.

\section{Apoptosis assays}

Cell monolayers were co-incubated with $C$. albicans (10 yeast cells/macrophage) for 5 or $15 \mathrm{~min}$ in RPMI medium at $37^{\circ} \mathrm{C}$, rinsed three times with the same medium and then either immediately processed for detection of apoptosis or re-incubated for up to $2 \mathrm{~h}$ before testing for apoptosis. The following tests were applied to the cell monolayers for detection of apoptosis: (1) optical microscopy analysis after staining with May-Grumwald-Giemsa; (2) fluorescence microscopy analysis of surface exposure of phosphatidylserine by means of the TACS annexin V-FITC kit (R\&D Systems, Minneapolis, MN, USA); and (3) in-situ terminal deoxynucleotidyl transferase nick end-labelling assay (TUNEL) by means of the TACS TdT kit (R\&D Systems). Either S. flexneri M90T or E. coli UEL $17\left(10^{8}\right.$ bacteria $\left./ \mathrm{ml}\right)$ were used as positive controls of apoptosis induction.

For analysis of DNA fragmentation, macrophages $\left(10^{6}\right.$ cells $/ \mathrm{ml}$, in RPMI 1640 medium) were allowed to adhere to round-bottomed wells (Costar plates) for $1 \mathrm{~h}$ at $37^{\circ} \mathrm{C}$, co-incubated for $15 \mathrm{~min}$ with $10^{7} \mathrm{C}$. albicans cells, and then rinsed and re-incubated for up to $4 \mathrm{~h}$. DNA of these cells was extracted by the phenolchloroform method [20], fractionated in agarose $1 \%$ gel in Tris-borate-EDTA buffer (c. 7-12 $\mu \mathrm{g}$ of DNA per lane), stained with ethidium bromide and photographed on an Image Master (Pharmacia).

\section{Staining by propidium iodide}

Macrophages co-incubated with $C$. albicans for $15 \mathrm{~min}$, according to a protocol similar to the one described above, were re-incubated for up to $2 \mathrm{~h}$ and then stained with propidium iodide (TACS annexin V-FITC kit).

\section{Results}

Morphology of macrophages and formation of germ-tubes by $C$. albicans CR1

Macrophages displayed several phagocytosed yeast cells contained within clearly delimited vesicles $15-$ 30 min (Fig. 1a) after their initial exposure to $C$. albicans CR1, and many of them had distorted nuclei and peripheral nuclear condensation. After $60 \mathrm{~min}, C$. albicans CR1 had frequently formed germ-tubes inside macrophages (Fig. 1b); after 120 min abundant hyphae had formed and the number of cells that remained attached to the glass slides was reduced. Macrophages co-incubated with $C$. albicans 577 rarely had germtubes in their interior after $60 \mathrm{~min}$ (Fig. 1c) and after $120 \mathrm{~min}$ no hyphae had formed around them. Both heat-inactivated $C$. albicans $\mathrm{CR} 1$ and viable $C$. albicans FCF14 were phagocytosed, but neither caused 

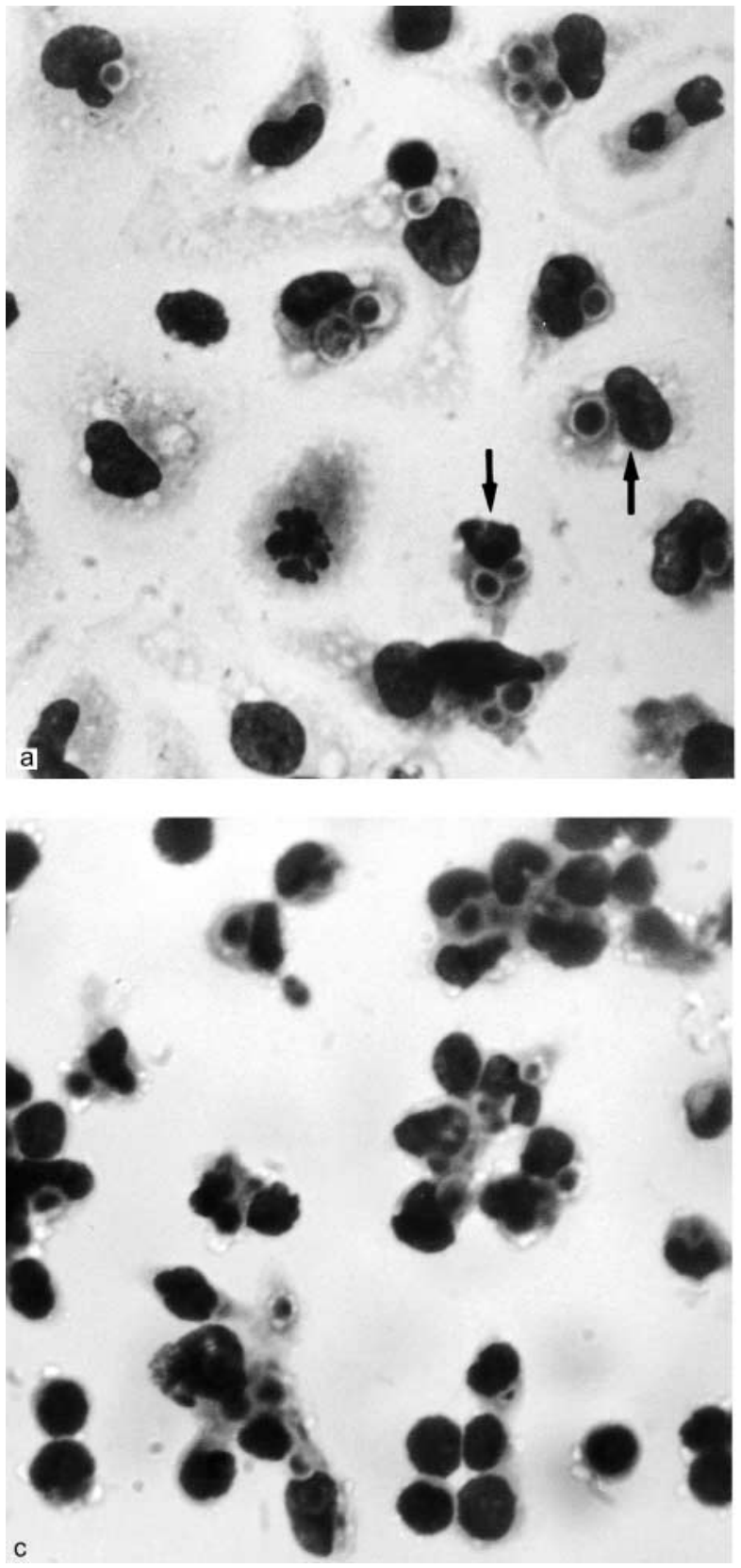

evident peripheral condensations in the nuclei of the cells that phagocytosed them, for up to $2 \mathrm{~h}$ of observation, and the latter strain did not form germtubes inside macrophages.

\section{Binding of annexin V-FITC to macrophages}

Several macrophages in each field bound annexin VFITC after co-incubation with $C$. albicans CR1 for 5 min (Fig. 2a), although many macrophages had not yet phagocytosed any yeast cells after that short period of incubation. After $15 \mathrm{~min}$, many macrophages coincubated with $C$. albicans CR1 were labelled by annexin V-FITC (Fig. 2b), whereas macrophages coincubated with $C$. albicans 577 were not labelled after that period (Fig. 2c) or after further incubation for up to $2 \mathrm{~h}$. Macrophages that phagocytosed heat-inactivated C. albicans CR1 did not bind annexin V-FITC, although several macrophages contained a large num-

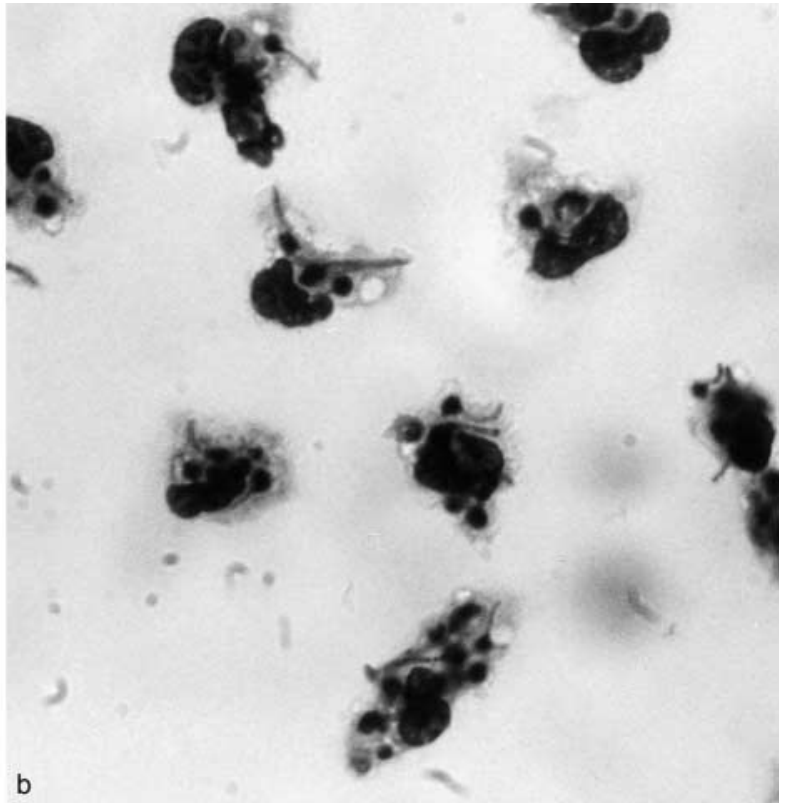

Fig. 1. Morphology of murine peritoneal macrophages coincubated with Candida albicans. (a) Macrophages co-incubated with $C$. albicans CR1 for 15 min. (b) Macrophages coincubated with $C$. albicans CR1 for $15 \mathrm{~min}$, rinsed and reincubated for $60 \mathrm{~min}$. (c) Macrophages co-incubated with $C$. albicans 577 for $15 \mathrm{~min}$, rinsed and re-incubated for $60 \mathrm{~min}$. Representative results of at least three independent experiments. May-Grumwald-Giemsa staining; magnification $800 \times$.

ber of ingested yeast cells after incubation for $15 \mathrm{~min}$. Macrophages co-incubated with C. albicans FCF-14 for periods of up to $2 \mathrm{~h}$ were not significantly labelled by annexin V-FITC.

\section{Macrophage labelling by propidium iodide}

Macrophages incubated with $C$. albicans CR1 became progressively permeable to propidium iodide. After exposure to $C$. albicans CR1 for $15 \mathrm{~min}$, few macrophages were labelled by propidium iodide, but after 60 min almost all macrophages in each field were labelled by both propidium iodide and annexin V-FITC (Fig. 3a); yeast cells unstained by propidium iodide could be seen inside macrophages. This increase in macrophage permeability seemed to occur mostly from $30 \mathrm{~min}$ (Fig. 3b) to $60 \mathrm{~min}$ (Fig. 3c) after exposure to C. albicans CR1, as shown by cells labelled only with propidium iodide. Co-incubation of macrophages with $C$. albicans 

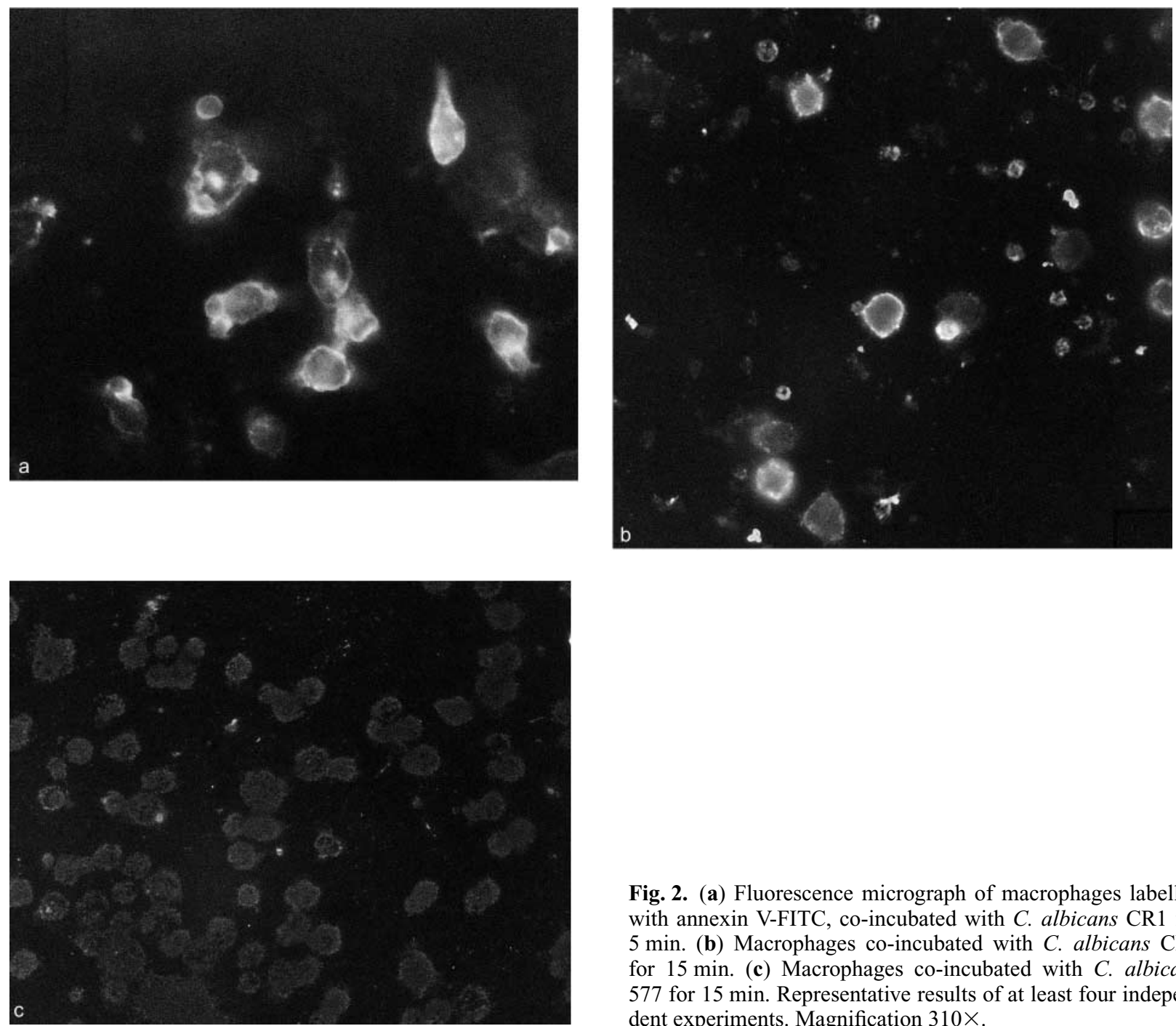

Fig. 2. (a) Fluorescence micrograph of macrophages labelled with annexin V-FITC, co-incubated with $C$. albicans CR1 for 5 min. (b) Macrophages co-incubated with C. albicans CR1 for 15 min. (c) Macrophages co-incubated with C. albicans 577 for $15 \mathrm{~min}$. Representative results of at least four independent experiments. Magnification $310 \times$.

577 did not cause a similar increase in permeability after 60 min. Macrophages were swollen (Fig. 3d) and entangled in the growing hyphae (Fig. 3e) $120 \mathrm{~min}$ after their initial exposure to $C$. albicans CR1. Co-incubation of macrophages with either heat-inactivated C. albicans CR1 or C. albicans FCF14 did not cause an increase in their permeability to propidium iodide similar to that caused by co-incubation with $C$. albicans CR1.

Effect of pepstatin-A on macrophage labelling by annexin V-FITC and propidium iodide

Macrophages co-incubated with pepstatin-A for $20 \mathrm{~min}$ were not labelled by annexin V-FITC after coincubation with $C$. albicans CR1 for $15 \mathrm{~min}$, and were rarely labelled after $30 \mathrm{~min}$. These macrophages became permeable to propidium iodide from 30 to $60 \mathrm{~min}$ after initial exposure to C. albicans CR1.

\section{TUNEL assays and DNA fragmentation}

Macrophages co-incubated with $C$. albicans CR1 did not bind the streptavidin-FITC conjugate in TUNEL assays performed 60 or $120 \mathrm{~min}$ (Fig. 4a) after their initial exposure to the yeast; TUNEL assays performed

at 5,15 or $30 \mathrm{~min}$ were also negative. Macrophages treated with TACS-nuclease (positive control), in each experiment, were labelled after incubation for $60 \mathrm{~min}$ (Fig. 4b) or $120 \mathrm{~min}$, and macrophages infected with $S$. flexneri were labelled after incubation for $4 \mathrm{~h}$. Agarose gel electrophoresis of DNA extracted from macrophages incubated with $C$. albicans CR1 did not show the ladder pattern of DNA degradation characteristic of apoptosis at any of the incubation periods tested $(5,15$, $30,60,120$ and $240 \mathrm{~min}$ ), whereas DNA from the positive control obtained by co-incubation with $S$. flexneri showed the apoptotic pattern.

\section{Discussion}

Several studies have shown that strains of $C$. albicans isolated from $\mathrm{HIV}$-infected individuals had increased expression of virulence traits such as proteinase production, adherence, resistance to antifungal drugs and phenotypic variation [21-23]; these observations support the conclusion that more virulent strains are either selected from commensal strains or replace them concomitantly with the lowering of the host's cellular defences. In this study, a strain of $C$. albicans (CR1) 

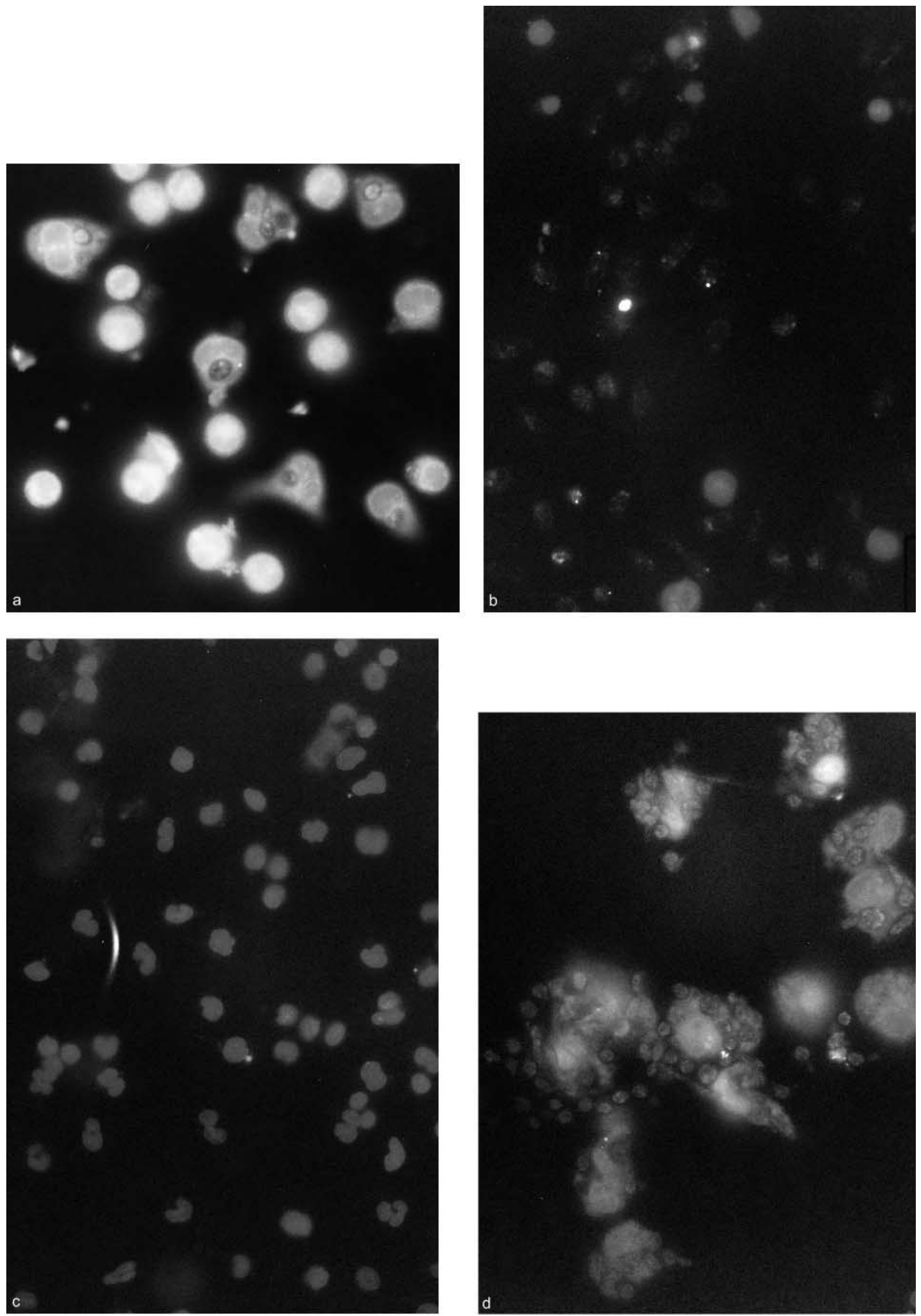

Fig. 3. (Continued on next page) 


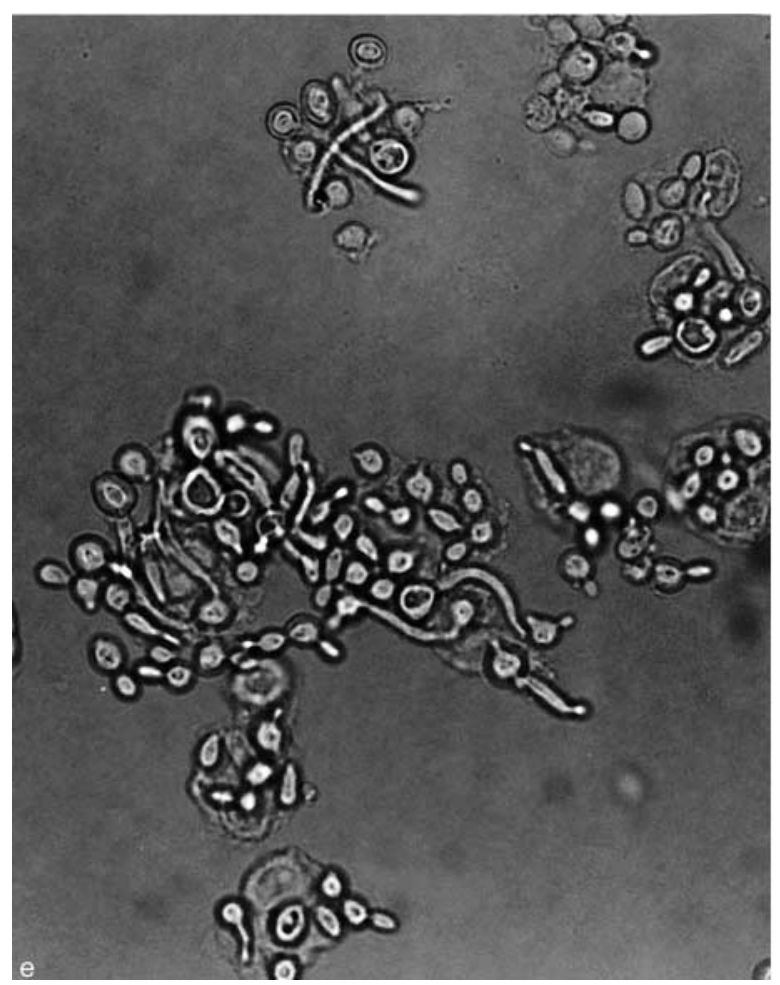

Fig. 3. Fluorescence micrographs of macrophages labelled with propidium iodide and annexin V-FITC. (a) Macrophages co-incubated with $C$. albicans CR1 for 15 min rinsed and reincubated to complete $60 \mathrm{~min}$ and labelled with propidium iodide and annexin V-FITC; (b) macrophages co-incubated with $C$. albicans $\mathrm{CR} 1$ for $15 \mathrm{~min}$, rinsed and re-incubated to complete $30 \mathrm{~min}$ or (c) $60 \mathrm{~min}$, and labelled with propidium iodide. (d) Macrophages labelled with propidium iodide 120 min after their initial exposure to C. albicans CR1, and (e) brightfield image from the same field as (d). Representative results of at least three independent experiments. Magnification: a, b, 320×; c, $360 \times$; d, e, $900 \times$.
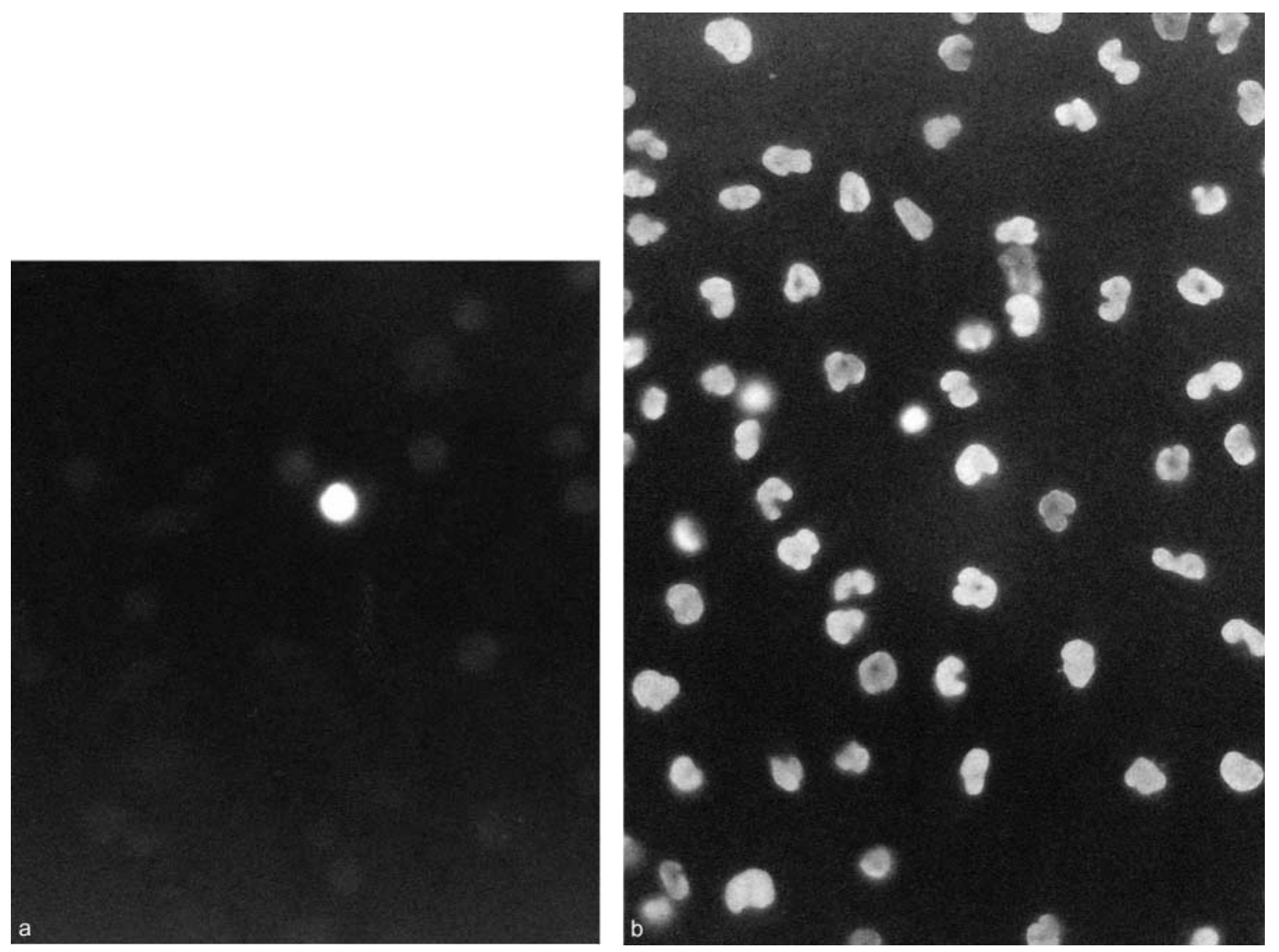

Fig. 4. (a) Fluorescence micrograph of TUNEL reactions applied to macrophages $120 \mathrm{~min}$ after their initial exposure to $C$. albicans CR1. Magnification $400 \times$. (b) Macrophages treated with nuclease (positive control) and tested by TUNEL after incubation for $60 \mathrm{~min}$. Representative results of at least five independent experiments. Magnification $370 \times$. 
isolated from an HIV-infected person induced membrane exposure of phosphatidylserine by murine macrophages after co-incubation for only 5 to $15 \mathrm{~min}$, which suggests that this strain exploits induction of apoptosis as a virulence factor. Although these macrophages had many phagocytosed yeast cells within clearly delimited vesicles $15-30 \mathrm{~min}$ after exposure to C. albicans CR1, after 1-2 h germ-tubes and hyphae developed and the macrophages were either permeable to propidium iodide or completely lysed. Neither heatinactivated C. albicans CR1 nor viable C. albicans 577 induced exposure of phosphatidylserine on the macrophage membranes, as judged from the absence of annexin V-FITC binding. Moreover, macrophages that phagocytosed heat-inactivated $C$. albicans CR1 remained impermeable to propidium iodide for up to $2 \mathrm{~h}$ of observation and did not present signs of chromatin condensation. C. albicans 577 rarely formed germtubes inside macrophages. Although these macrophage monolayers were better preserved, some cells were permeable to propidium iodide after incubation for 1$2 \mathrm{~h}$. These in-vitro observations support the inference that $C$. albicans CR1 uses induction of apoptosis and switching to germ-tubes inside macrophages as a mechanism to evade the host's defences. However, to estimate whether this is a trait common in virulent strains, the ability of a large number of Candida strains to induce apoptosis and switch to germ-tubes inside host cells should be evaluated.

Secretion of aspartyl proteinases is considered to be an important virulence trait of C. albicans, and strain CR1 produces proteases and phospholipases, as detected on agar plates (unpublished results). Macrophages preincubated with the proteinase inhibitor pepstatin A had reduced binding of annexin after exposure to $C$. albicans CR1, although pepstatin A did not protect those macrophages from becoming permeable to propidium iodide $1-2 \mathrm{~h}$ after being exposed to the yeast. This suggests that secreted proteinases participate in the triggering of phosphatidylserine exposure, but other factors are responsible for cell necrosis. Apparently in agreement with this conclusion, $C$. albicans FCF14, which is deficient in proteinases and phospholipases, did not induce exposure of phosphatidylserine nor cause necrosis of macrophages for up to $2 \mathrm{~h}$ of observation. However, this conclusion partly contradicts some previous results, because C. albicans 577 produces proteinases and phospholipases and did not induce exposure of phosphatidylserine. Quantitative differences in the expression of virulence factors by those strains might be responsible for this apparent contradiction, as $C$. albicans CR1 expresses about twice as much catalase activity as C. albicans 577 (unpublished results), which would be expected to confer better protection to the former strain against hydrogen peroxide released by phagocytes. However, the present data do not exclude the possible existence of qualitative differences between the expression of virulence factors by these two strains.
Both exposure of phosphatidylserine and internucleosomal degradation of DNA are hallmarks of apoptosis. However, repeated TUNEL tests conducted at several time intervals after exposure of macrophages to $C$. albicans CR1 did not show any significant labelling, indicating the absence of degraded DNA nick ends, although the positive controls always confirmed that the reaction worked properly. Absence of the DNA degradation typical of apoptosis was also repeatedly confirmed by agarose gel electrophoresis. These results might indicate that apoptosis is initially triggered and then interrupted by further interactions between the phagocytosed yeasts and the macrophage. Another possible explanation is that $C$. albicans CR1 induces apoptosis through the recently discovered caspaseindependent pathway of programmed cell death, in which apoptosis-inducing factor (AIF) released from the mitochondria acts directly on the cell nucleus and leads to degradation of DNA into large fragments of $c$. $50 \mathrm{~kb}[24,25]$. As AIF causes nuclear chromatin condensation, this last explanation also encompasses the initial observation that macrophages showed chromatin condensation $30 \mathrm{~min}$ after exposure to $C$. albicans CR1.

Several studies have demonstrated induction of apoptosis of macrophages and other cell lines a few hours after bacterial infection in vitro [1-7], but in this study exposure of phosphatidylserine by macrophages was observed within a few minutes of their contact with $C$. albicans CR1. However, the macrophages did not progress into the well-known sequence of apoptotic changes and, after 1-2 $\mathrm{h}$ almost all of them were either necrotic or lysed. This suggests that apoptotic cells, which have their mitochondrial functions impaired, might provide an adequate temporary environment for the switching of yeast cells to germ-tubes. If some strains of Candida are able to induce apoptosis of macrophages in vivo, yeast strains that readily switch to germ-tubes inside those apoptotic cells would have a competitive advantage, because germ-tubes released by cell lysis are more resistant than yeast cells to further attack by incoming macrophages.

\section{References}

1. Zychlinsky A, Prevost MC, Sansonetti PJ. Shigella flexneri induces apoptosis in infected macrophages. Nature 1992; 358: $167-169$.

2. Khelef N, Zychlinky A, Guiso N. Bordetella pertussis induces apoptosis in macrophages: role of adenylate cyclase-hemolysin. Infect Immun 1993; 61: 4064-4071.

3. Baran J, Guzik K, Hryniewicz W, Ernst M, Flad HD, Pryjma J. Apoptosis of monocytes and prolonged survival of granulocytes as a result of phagocytosis of bacteria. Infect Immun 1996; 64: 4242-4248.

4. Monack DM, Raupach B, Hromockyj AE, Falkow S. Salmonella typhimurium invasion induces apoptosis in infected macrophages. Proc Natl Acad Sci USA 1996; 93: 9833-9838.

5. Ruckdeschel K, Roggenkamp A, Lafont V, Mangeat P, Heesemann J, Rouot B. Interaction of Yersinia enterocolitica with macrophages leads to macrophage cell death through apoptosis. Infect Immun 1997; 65: 4813-4821. 
6. Rodrigues VS, Vidotto MC, Felipe I, Santos DS, Gaziri LCJ. Apoptosis of murine peritoneal macrophages induced by an avian pathogenic strain of Escherichia coli. FEMS Microbiol Lett 1999; 179: 73-78.

7. Jendrossek V, Grassmé H, Mueller I, Lang F, Gulbins E. Pseudomonas aeruginosa-induced apoptosis involves mitochondria and stress-activated protein kinases. Infect Immun 2001; 69: 2675-2683.

8. Zychlinsky A, Kenny B, Ménard R, Prévost MC, Holland IB Sansonetti PJ. Ipa B mediates macrophage apoptosis induced by Shigella flexneri. Mol Microbiol 1994; 11: 619-627.

9. Chen LM, Kaniga K, Galán JE. Salmonella spp. are cytotoxic for cultured macrophages. Mol Microbiol 1996; 21: 11011115 .

10. Chen Y, Smith MR, Thirumalai K, Zychlinsky A. A bacterial invasin induces macrophage apoptosis by binding directly to ICE. $E M B O J$ 1996; 15: 3853-3860.

11. Jacotot E, Ravagnan L, Loeffler M et al. The HIV-1 viral protein $\mathrm{R}$ induces apoptosis via a direct effect on the mitochondrial permeability transition pore. J Exp Med 2000; 191: 33-46.

12. Miura T, Nishikawa S, Sasaki $\mathrm{S}$ et al. Role of endogenous cytokines in liver apoptosis of mice in lethal Listeria monocytogenes infection. FEMS Immunol Med Microbiol 2000; 28: $335-341$.

13. Perfettini JL, Darville T, Gachelin G et al. Effect of Chlamydia trachomatis infection and subsequent tumor necrosis factor alpha secretion on apoptosis in the murine genital tract. Infect Immun 2000; 68: 2237-2244.

14. Rotstein D, Parodo J, Taneja R, Marshall JC. Phagocytosis of Candida albicans induces apoptosis of human neutrophils. Shock 2000; 14: 278-283.

15. Heidenreich S, Otte B, Lang D, Schmidt M. Infection by Candida albicans inhibits apoptosis of human monocytes and monocytic U937 cells. J Leukoc Biol 1996; 60: 737-743.
16. Sweeney JF, Nguyen PK, Omann GM, Hinshaw DB. Autocrine-paracrine modulation of polymorphonuclear leukocyte survival after exposure to Candida albicans. Shock 1998; 9: $146-152$.

17. Pacheco-Soares C, Gaziri LCJ, Loyola W, Felipe I. Phagocytosis of enteropathogenic Escherichia coli and Candida albicans by lectin-like receptors. Braz J Med Biol Res 1992; 25: $1015-1024$.

18. Gaziri G, Gaziri LCJ, Kikuchi R, Scanavacca J, Felipe I. Phagocytosis of Candida albicans by concanavalin-A activated peritoneal phagocytes. Med Mycol 1999; 37: 195-200.

19. Moresco TR, Gaziri LCJ, Yasumoto Y, Felipe I. Phagocytic and candidacidal activities of macrophages from lactating and adult mice pretreated with concanavalin-A. Med Mycol (in press)

20. Davis LG, Kuehl WM, Battey JF. Basic methods in molecular biology. Norwalk, CT, Appleton and Lange. 1994: 16-21.

21. Sweet SP, Cookson S, Challacombe SJ. Candida albicans isolates from HIV-infected and AIDS patients exhibit enhanced adherence to epithelial cells. J Med Microbiol 1995; 43: 452457.

22. de Bernardis F, Mondello F, Scaravelli G et al. High aspartyl proteinase production and vaginitis in human immunodeficiency virus-infected women. J Clin Microbiol 1999; 37: 1376-1380.

23. Vargas K, Messer AS, Pfaller M et al. Elevated phenotypic switching and drug resistance of Candida albicans from human immunodeficiency virus-positive individuals prior to first thrush episode. J Clin Microbiol 2000; 38: 3595-3607.

24. Daugas E, Nochy D, Ravagnan L et al. Apoptosis-inducing factor (AIF): a ubiquitous mitochondrial oxidoreductase involved in apoptosis. FEBS Lett 2000; 476: 118-123.

25. Joza N, Susin SA, Daugas E et al. Essential role of the mitochondrial apoptosis-inducing factor in programmed cell death. Nature 2001; 410: 549-554. 